\title{
Behaviour of 4-[4-methoxy-3-methylphenyl]-4-oxobutenoic acid towards nitrogen-containing nucleophiles
}

\author{
SAHAR SAID EL-SAKKA*, MOHAMED HELMY SOLIMAN and \\ ROKAIA SAFWAT ABDULLAH \\ Suez University, Faculty of Science, Chemistry Department, Suez, Egypt \\ e-mail: saharelsakka@hotmail.com
}

MS received 16 March 2014; revised 17 June 2014; accepted 18 June 2014

\begin{abstract}
A series of novel amino acid derivatives has been synthesized by the reaction of 4-[4-methoxy3-methylphenyl]-4-oxobutenoic acid with primary and secondary amines. The treatment of amino acids with hydrazine afforded pyridazine. Phenylhydrazone was obtained from the reaction of the acid with phenyl hydrazine in ethanol. On the other hand, the acid underwent heterocyclization upon the treatment with 2-aminopyridine, $o$-phenylenediamine, aryldithiocarbamates and thiourea derivatives to give the corresponding pyridopyrimidine, quinoxalone, 2-thioxo-1,3-thiazole and 4-hydroxy-1,3-thiazole, respectively. The thiazolopyridazine derivatives were obtained from the reaction of 4-hydroxy-1,3-thiazole with hydrazine and phenylhydrazine, respectively. The behaviour of the 4-hydroxy-1,3-thiazole toward acetic anhydride and bromine was also studied. The proposed structures of the products were based on microanalytical and spectroscopic data. Some of the synthesized compounds also exhibited anti-microbial activities.
\end{abstract}

Keywords. $\quad \beta$-aroylacrylic acid; amino acid; pyridazine; quinoxalone; thiazole; thiazolopyridazine; AzaMichael addition

\section{Introduction}

It was found that $\beta$-Aroylacrylic acids and their derivatives showed effective biological activities including antibacterial activity, ${ }^{1-4}$ anti-ulcer and cytoprotective properties, ${ }^{5}$ kynurenine-3-hydroxylase, ${ }^{6}$ and antiproliferative activity toward Human cervix carcinoma (HeLa). ${ }^{7,8}$ Besides their broad spectrum of biological activity, $\beta$-aroylacrylic acids are versatile and convenient precursors which have been extensively utilized in the synthesis of a wide variety of heterocyclic compounds. ${ }^{9-12}$ The $\alpha, \beta$-unsaturated carbonyl moiety of these compounds is a favorable unit for addition of different nucleophiles which occurs exclusively at the olefin electrophilic centre of the molecule and usually results in the formation of various nitrogen-containing heterocyclic compounds of different ring sizes with one or more heteroatom.

In this regard, the present work deals with the reactivity of 4-[4-methoxy-3-methylphenyl]-4-oxobutenoic acid towards some nucleophilic reagents in order to synthesize a novel series of heterocyclic compounds such as quinaxoline, thiazole, thiazolopyridazine, pyridazine bearing 4-methoxy-3-methylphenyl moiety for biological evaluation.

\footnotetext{
*For correspondence
}

\section{Experimental}

All melting points reported are uncorrected and determined by the open capillary tube method on a Gallen Kamp melting point apparatus. ${ }^{1} \mathrm{H}-\mathrm{NMR}$ spectra were measured on Bruker (200 MHz) with DMSO-d6 as the solvent and TMS was used as internal standard (chemical shifts are expressed as $\delta$, ppm), IR spectra were recorded on a Perking Elmer 1430 ratio recording infrared spectrophotometer with CDS data station using $\mathrm{KBr}$ Wafer technique and Mass spectra were measured on a GC-MSQP 1000EX Shimadzu at microanalytical laboratory, Cairo University, Cairo, Egypt.

\subsection{General procedure for the synthesis of 2- substitutedamino-4-[4-methoxy-3-methylphenyl]-4- oxobutanoic acids (2a-e)}

To a stirred solution of 4-[4-methoxy-3-methyl]-4oxobutenoic acid (1) $(0.01 \mathrm{~mol})$ in benzene $(40 \mathrm{~mL})$ was added the appropriate amines, namely benzyl amine; cyclohexyl amine; morpholine; or/ and piperidine $(0.01 \mathrm{~mol})$. The reaction mixture was stirred at room temperature for $2 \mathrm{hrs}$., then kept overnight, the resultant precipitate was filtered off, washed with 
benzene and crystallized from suitable solvent to give (2a-e) as white solid.

2.1a 2-benzylamino-4-[4-methoxy-3-methylphenyl]4-oxobutanoic acid (2a): From benzene/ethanol; M.p. $170^{\circ} \mathrm{C}$; Anal. Calcd. for $\mathrm{C}_{19} \mathrm{H}_{21} \mathrm{NO}_{4}$ (327.38): C, 69.71; H, 6.47; N, 4.28. Found: C, 70.00; H, 6.67; N, 4.40; IR $\left(\mathrm{KBr}, v, \mathrm{~cm}^{-1}\right): 3436(\mathrm{OH}), 1668(\mathrm{C}=\mathrm{O}) .{ }^{1} \mathrm{H}-\mathrm{NMR}$ (DMSO- $\left.d_{6}, \delta / \mathrm{ppm}\right): 2.18$ (3H, s, $\left.\mathrm{CH}_{3} \mathrm{Ar}\right), 3.31-3.36$ (2H, m, $\left.\underline{\mathrm{CH}}_{2}-\mathrm{CH}\right), 3.61-3.64\left(\mathrm{H}, \mathrm{m}, \underline{\mathrm{CH}}-\mathrm{CH}_{2}\right), 3.86$ $\left(3 \mathrm{H}, \mathrm{s}, \mathrm{OCH}_{3}\right), 3.88\left(2 \mathrm{H}, \mathrm{s}, \mathrm{N}-\mathrm{CH}_{2} \mathrm{Ph}\right), 6.65-7.85$ $(10 \mathrm{H}, \mathrm{m}, \mathrm{Ar}-\mathrm{H}, \mathrm{NH}$ and $\mathrm{OH}) ; \mathrm{MS}(m / z, 327(0.03 \%))$ $\left(\mathrm{M}^{+}\right)$.

2.1b 2-cyclohexylamino-4-[4-methoxy-3-methylphenl]4-oxobutanoic acid (2b): From toluene, M.p. $180^{\circ} \mathrm{C}$; Anal. Calcd. for $\mathrm{C}_{18} \mathrm{H}_{25} \mathrm{NO}_{4}$ (319.40): C, 67.69; H, 7.89; N, 4.39. Found: C, 67.72; H, 7.75; N, 4.51; IR $\left(\mathrm{KBr}, v, \mathrm{~cm}^{-1}\right): 3438(\mathrm{OH}), 3337(\mathrm{NH}), 2929(\mathrm{CH}-$ aliph.), $1675(\mathrm{C}=\mathrm{O}) ;{ }^{1} \mathrm{H}-\mathrm{NMR}$ (DMSO- $\left.d_{6}, \delta \mathrm{ppm}\right)$ : 1.07-1.69 $(11 \mathrm{H}, \mathrm{m}$, cyclohexyl group), $2.20(3 \mathrm{H}, \mathrm{s}$, $\left.\mathrm{CH}_{3} \mathrm{Ar}\right), 2.86-2.90\left(2 \mathrm{H}, \mathrm{m}, \underline{\mathrm{CH}}_{2}-\mathrm{CH}\right), 3.36-3.40(\mathrm{H}$, $\left.\mathrm{m}, \underline{\mathrm{CH}}-\mathrm{CH}_{2}\right), 3.88\left(3 \mathrm{H}, \mathrm{s}, \mathrm{OCH}_{3}\right), 6.64-7.86(5 \mathrm{H}, \mathrm{m}$, $\mathrm{Ar}-\mathrm{H}, \mathrm{NH}$ and $\mathrm{OH})$.

2.1c 2-(2-hydroxyethylamino)-4-[4-methoxy-3methylphenyl]-4-oxobutanoic acid (2c): From butanol, M.p. $172^{\circ}$ C. Anal. Calcd. for $\mathrm{C}_{14} \mathrm{H}_{19} \mathrm{NO}_{5}$ (281.30): C, 59.78; H, 6.81; N, 4.98., Found: C, 59.80; H, 7.03; N, 5.10; IR (KBr, $\left.v, \mathrm{~cm}^{-1}\right)$ : $3275(\mathrm{NH}), 2958$ (CH-aliph), $1670(\mathrm{C}=\mathrm{O}) ;{ }^{1} \mathrm{H}-\mathrm{NMR}$ (DMSO- $\left.d_{6}, \delta \mathrm{ppm}\right): 2.16$ (3H, s, $\left.\mathrm{CH}_{3} \mathrm{Ar}\right), 2.83-2.98$ (4H, 2t, $\left.\mathrm{CH}_{2}-\mathrm{CH}_{2}\right), 3.41-$ $3.47\left(2 \mathrm{H}, \mathrm{m}, \underline{\mathrm{CH}}_{2}-\mathrm{CH}\right), 3.56-3.69\left(\mathrm{H}, \mathrm{m}, \underline{\mathrm{CH}}-\mathrm{CH}_{2}\right)$, $3.88\left(3 \mathrm{H}, \mathrm{s}, \mathrm{OCH}_{3}\right), 6.63-7.86(6 \mathrm{H}, \mathrm{m}, \mathrm{Ar}-\mathrm{H}, \mathrm{NH}$ and $\mathrm{OH})$.

2.1d 4-[4-methoxy-3-methylphenyl]-2-piperidino-4oxobutanoic acid (2d): From ethanol, M.p. $156^{\circ}$ C. Anal. Calcd. for $\mathrm{C}_{17} \mathrm{H}_{23} \mathrm{NO}_{4}$ (305.37): C, 66.86; H, 7.59; N, 4.59. Found: C, 66.95; H, 7.78; N, 4.38; IR $\left(\mathrm{KBr}, v, \mathrm{~cm}^{-1}\right): 3437(\mathrm{OH}), 2941$ (CH-aliph.), 1675 $(\mathrm{C}=\mathrm{O}) ;{ }^{1} \mathrm{H}-\mathrm{NMR}$ (DMSO- $\left.d_{6}, \delta \mathrm{ppm}\right): 1.37-1.63(6 \mathrm{H}$, $\mathrm{m}$, piperidine), $2.19\left(3 \mathrm{H}, \mathrm{s}, \mathrm{CH}_{3} \mathrm{Ar}\right), 2.48-2.77(4 \mathrm{H}$, $\mathrm{m}$, piperidine), 2.93-2.96 (2H, m, $\left.\underline{\mathrm{CH}}_{2}-\mathrm{CH}\right), 3.71-3.77$ $\left(\mathrm{H}, \mathrm{m} \underline{\mathrm{CH}}-\mathrm{CH}_{2}\right), 3.88\left(3 \mathrm{H}, \mathrm{s}, \mathrm{OCH}_{3}\right), 6.65-7.85(5 \mathrm{H}$, $\mathrm{m}, \mathrm{Ar}-\mathrm{H}$ and $\mathrm{OH}) ; \mathrm{MS}(\mathrm{m} / z, 305(5.7 \%))\left(\mathrm{M}^{+}\right)$.

2.1e 4-[4-methoxy-3-methylphenyl]-2-morpholino-4oxobutanoic acid (2e): From toluene, M.p. $172^{\circ} \mathrm{C}$. Anal. Calcd. for $\mathrm{C}_{16} \mathrm{H}_{21} \mathrm{NO}_{5}$ (307.34): C, 62.53; $\mathrm{H}$, 6.89; N, 4.56. Found: C, 62.70; H, 7.02; N, 4.71; IR
$\left(\mathrm{KBr}, v, \mathrm{~cm}^{-1}\right): 3433(\mathrm{OH}), 2973(\mathrm{CH}$-aliph$), 1675$ $(\mathrm{C}=\mathrm{O}) ;{ }^{1} \mathrm{H}-\mathrm{NMR}$ (DMSO- $\left.d_{6}, \delta \mathrm{ppm}\right): 2.19(3 \mathrm{H}, \mathrm{s}$, $\left.\mathrm{CH}_{3} \mathrm{Ar}\right), 2.34-2.83\left(2 \mathrm{H}, \mathrm{m}, \underline{\mathrm{CH}}_{2}-\mathrm{CH}\right), 3.04-3.10(\mathrm{H}$, $\left.\mathrm{m} \underline{\mathrm{CH}}-\mathrm{CH}_{2}\right), 2.43-2.51$ and $3.49-3.87$ (8H, 2m, morpholin), $3.89\left(3 \mathrm{H}, \mathrm{s}, \mathrm{OCH}_{3}\right), 7.02-7.89(4 \mathrm{H}, \mathrm{m}, \mathrm{Ar}-\mathrm{H}$ and $\mathrm{OH}) ; \mathrm{MS}(m / z, 306(16.4 \%))\left(\mathrm{M}^{+}-1\right)$.

\subsection{Formation of pyridazinone (3)}

Hydrazine hydrate $80 \%$ ( $0.015 \mathrm{~mol})$ was added to a solution of 2-substituted amino-butanoic acids (2a) or $(2 b)$ or $(2 \mathrm{c})$ or $(2 \mathrm{~d})(0.01 \mathrm{~mol})$ in ethanol $(30 \mathrm{~mL})$ and the reaction mixture was heated under reflux for $6 \mathrm{hrs}$., after concentration and cooling, the product obtained was collected and crystallized from ethanol to give pyridazinone (3) as white crystals with M.p. $142^{\circ} \mathrm{C}$. Anal. Calcd. for $\mathrm{C}_{12} \mathrm{H}_{12} \mathrm{~N}_{2} \mathrm{O}_{2}$ (216.23): C, 66.65; H, 5.59; N, 12.95. Found: C, 66.83; H, 5.79; N, 12.87; IR (KBr, $\left.v, \mathrm{~cm}^{-1}\right): 3443(\mathrm{NH})$ or $(\mathrm{OH}), 1668(\mathrm{C}=\mathrm{O}) ;{ }^{1} \mathrm{H}-\mathrm{NMR}$ $\left(\mathrm{CDCl}_{3}-d_{6}, \delta \mathrm{ppm}\right): 2.28\left(3 \mathrm{H}, \mathrm{s}, \mathrm{CH}_{3}\right), 3.89(3 \mathrm{H}, \mathrm{s}$, $\left.\mathrm{CH}_{3} \mathrm{O}\right), 6.88-6.91(1 \mathrm{H}, \mathrm{d}, \mathrm{H}-5$ pyridazinone, $J=8.23$ $\mathrm{Hz}), 7.01-7.05(1 \mathrm{H}, \mathrm{d}, \mathrm{H}-6$ benzene ring, $J=9.85$ $\mathrm{Hz}), 7.26(1 \mathrm{H}, \mathrm{s}, \mathrm{H}-2$ benzene ring), 7.55-7.56 $(1 \mathrm{H}, \mathrm{d}$, $\mathrm{H}-5$ benzene ring, $J=9.80 \mathrm{~Hz}), 7.71-7.75(1 \mathrm{H}, \mathrm{d}, \mathrm{H}-$ 4 pyridazinone, $J=9.75 \mathrm{~Hz}), 10.9(1 \mathrm{H}$, broad, $\mathrm{N}-\mathrm{H}$ pyridazine); $\operatorname{MS}(m / z, 216(100 \%))\left(\mathrm{M}^{+}\right)$.

\subsection{Synthesis of 4-[4-methoxy-3-methylphenyl]-4-} oxobutenoic acid phenyl hydrazone (4)

To a solution of the acid (1) $(0.01 \mathrm{~mol})$ in ethanol (20 $\mathrm{mL})$, phenyl hydrazine $(0.01 \mathrm{~mol})$ was added and the reaction mixture was heated under water bath for $1 / 2$ hr., the solid product separated after cooling was filtered off and crystallized from ethanol to give orange crystals (4) M.p. $209^{\circ}$ C. Anal. Calcd. for $\mathrm{C}_{18} \mathrm{H}_{18} \mathrm{~N}_{2} \mathrm{O}_{3}$ (310.35): C, 69.66; H, 5.85; N, 9.03. Found: C, 69.83; H, 5.90; N, 9.14; IR (KBr, v, cm $\left.{ }^{-1}\right): 3587(\mathrm{OH}), 3294(\mathrm{NH})$, $1679(\mathrm{C}=\mathrm{O}) ;{ }^{1} \mathrm{H}-\mathrm{NMR}$ (DMSO- $\left.d_{6}, \delta \mathrm{ppm}\right): 2.21(3 \mathrm{H}, \mathrm{s}$, $\left.\mathrm{CH}_{3} \mathrm{Ar}\right), 3.87\left(3 \mathrm{H}, \mathrm{s}, \mathrm{OCH}_{3}\right), 5.29-5.34(\mathrm{H}, \mathrm{d}, \mathrm{CH}=\underline{\mathrm{CH}}$, $J=15.9 \mathrm{~Hz}), 6.82-7.46(9 \mathrm{H}, \mathrm{m}, \mathrm{Ar}-\mathrm{H}$ and $\mathrm{CH}=\mathrm{CH})$, $9.35(\mathrm{H}, \mathrm{s}, \mathrm{NH}), 12.14(\mathrm{H}, \mathrm{s}, \mathrm{OH}) ; \mathrm{MS}(\mathrm{m} / z, 310$ $(19.6 \%))\left(\mathrm{M}^{+}\right)$.

\subsection{Synthesis of 4-[6-chlorobenzothiazol-2-ylimino]- 4-[4-methoxy-3-methylphenyl]-4-oxobutenoic (5)}

A mixture of the acid $(0.01 \mathrm{~mol})$ and 6-chloro-2aminobenzothiazol $(0.01 \mathrm{~mol})$ in ethanol $(20 \mathrm{~mL})$ was refluxed for $8 \mathrm{hrs}$. The solid product separated after cooling was filtered off and crystallized from 
ethanol to give the compound (5). Anal. Calcd. for $\mathrm{C}_{19} \mathrm{H}_{15} \mathrm{ClN}_{2} \mathrm{O}_{3} \mathrm{~S}$ (386.85): C, 58.99; H, 3.91; N, 7.24. Found: $\mathrm{C}, 59.02 ; \mathrm{H}, 3.77 ; \mathrm{N}, 7.31$. IR (KBr, v, $\left.\mathrm{cm}^{-1}\right)$ : $3300(\mathrm{OH}), 3172(\mathrm{NH}), 1652(\mathrm{C}=\mathrm{O}) ;{ }^{1} \mathrm{H}-\mathrm{NMR}$ (DMSO- $\left.d_{6}, \delta \mathrm{ppm}\right): 2.18\left(3 \mathrm{H}, \mathrm{s}, \mathrm{CH}_{3} \mathrm{Ar}\right), 3.89(3 \mathrm{H}, \mathrm{s}$, $\left.\mathrm{OCH}_{3}\right), 6.62-7.97(9 \mathrm{H}, \mathrm{m}, \mathrm{Ar}-\mathrm{H}, \underline{\mathrm{CH}}=\underline{\mathrm{CH}}$ and $\mathrm{OH})$.

2.5 Synthesis of 2-[4-methoxy-3-methylphenyl]-4Hpyrido[1,2-a]pyrimidine-4-carboxylic acid (6)

A mixture of the acid (1) (0.01 mol) and 2aminopyridine $(0.01 \mathrm{~mol})$ in ethanol $(40 \mathrm{~mL})$ was refluxed for 2 hrs., the product separated after cooling was filtered off, washed with cold ethanol and crystallized from ethanol to give yellow crystals of (6) with M.p. $145^{\circ} \mathrm{C}$. Anal. Calcd. for $\mathrm{C}_{17} \mathrm{H}_{16} \mathrm{~N}_{2} \mathrm{O}_{3}$ (296.32): C, 68.91; H, 5.44; N, 9.45. Found: C, 68.85; H, 5.53; N, 9.52; IR (KBr, $\left.v, \mathrm{~cm}^{-1}\right): 3451(\mathrm{OH}), 1649(\mathrm{C}=\mathrm{O}) ;{ }^{1} \mathrm{H}-$ NMR (DMSO- $d_{6}, \delta$ ppm): $2.14\left(3 \mathrm{H}, \mathrm{s}, \mathrm{CH}_{3} \mathrm{Ar}\right), 3.77$ $\left(3 \mathrm{H}, \mathrm{s}, \mathrm{OCH}_{3}\right), 4.82-4.83(\mathrm{H}, \mathrm{d}, \mathrm{CH}-\mathrm{COOH}), 6.17-7.55$ $(8 \mathrm{H}, \mathrm{m}, \mathrm{Ar}-\mathrm{H}) ; \mathrm{MS}(\mathrm{m} / z, 252(51.9 \%))\left(\mathrm{M}^{+}-\mathrm{CO}_{2}\right)$.

2.6 Synthesis of 3,4-dihydro-3-(2-(4-methoxy-3methylphenyl)-2-oxoethyl)-1H-quinoxalin-2-one (7)

A mixture of $o$-phenylenediamine $(0.005 \mathrm{~mol})$ and the acid (1) $(0.005 \mathrm{~mol})$ in ethanol $(15 \mathrm{~mL})$ was heated under reflux for $6 \mathrm{~h}$. The solid product obtained after cooling was filtered off and crystallized from ethanol to give the product (7) with M.p. $180^{\circ} \mathrm{C}$. Anal. Calcd. for $\mathrm{C}_{18} \mathrm{H}_{18} \mathrm{~N}_{2} \mathrm{O}_{3}$ (310.35): C, 69.66; H, 5.85; N, 9.03. Found: C, 69.66; H, 5.90; N, 8.91; IR $\left(\mathrm{KBr}, v, \mathrm{~cm}^{-1}\right)$ : $3369(\mathrm{NH}), 3196(\mathrm{NH}), 1688(\mathrm{C}=\mathrm{O}), 1663(\mathrm{C}=\mathrm{O}) ;{ }^{1} \mathrm{H}-$ NMR (DMSO- $d_{6}, \delta$ ppm): $2.20\left(3 \mathrm{H}, \mathrm{s}, \mathrm{CH}_{3} \mathrm{Ar}\right), 3.47-$ $3.45\left(2 \mathrm{H}, \mathrm{m}, \underline{\mathrm{CH}}_{2}-\mathrm{CH}\right), 3.89\left(3 \mathrm{H}, \mathrm{s}, \mathrm{OCH}_{3}\right), 4.30-4.38$ $\left(\mathrm{H}, \mathrm{m} \underline{\mathrm{CH}}-\mathrm{CH}_{2}\right), 2.63(\mathrm{H}, \mathrm{s}, \mathrm{NH}), 6.60-7.89(7 \mathrm{H}, \mathrm{m}$, Ar-H), 10.28 (H, s, NH); MS (m/z, $310(6.29 \%))\left(\mathrm{M}^{+}\right)$.

2.7 General procedure for the synthesis of 3-Aryl-5[2-(4-methoxy-3-methylphenyl)-2-oxoethyl]-2-thioxo1,3-thiazolidin-4-ones (8a-c)

To a stirred solution of the acid (1) (0.005 mol) in EtOH $(10 \mathrm{~mL})$, ammonium $\mathrm{N}$-aryldithiocarbamate $(0.005$ mol), namely ammonium N-benzyl dithiocarbamate; ammonium N-phenyldithiocarbamate, ammonium N-4methylphenyldithiocarbamate, was added portion wise and stirred at room temperature for $30 \mathrm{~min}$. Then, the mixture was acidified with conc. $\mathrm{HCl}(3 \mathrm{~mL})$, boiled for $5 \mathrm{~min}$ and left to cool. The precipitated solid was filtered off, washed with $\mathrm{H}_{2} \mathrm{O}$, air dried, and the crude product was crystallized from the proper solvent to give the product. 2.7a 5-[2-(4-methoxy-3-methylphenyl)-2-oxoethyl]-3phenyl-2-thioxo-1,3-thiazolidin-4-one (8a): From ethanol, M.p. $145^{\circ} \mathrm{C}$. Anal. Calcd. for $\mathrm{C}_{19} \mathrm{H}_{17} \mathrm{NO}_{3} \mathrm{~S}_{2}$ (371.46): C, 61.43; H, 4.61; N, 3.77. Found: C, 61.83; H, 4.58; $\mathrm{N}, 4.15$; IR $\left(\mathrm{KBr}, v, \mathrm{~cm}^{-1}\right): 1740(\mathrm{C}=\mathrm{O}), 1659(\mathrm{C}=\mathrm{O})$, $1254(\mathrm{C}=\mathrm{S}) ;{ }^{1} \mathrm{H}-\mathrm{NMR}$ (DMSO- $\left.d_{6}, \delta \mathrm{ppm}\right): 2.07(3 \mathrm{H}$, s, $\left.\mathrm{CH}_{3} \mathrm{Ar}\right), 3.91\left(3 \mathrm{H}, \mathrm{s}, \mathrm{OCH}_{3}\right), 3.94-4.17(2 \mathrm{H}, \mathrm{m}$, $\left.\underline{\mathrm{CH}}_{2}-\mathrm{CH}\right), 4.95-4.99\left(\mathrm{H}, \mathrm{m}, \underline{\mathrm{CH}}-\mathrm{CH}_{2}\right), 7.07-7.91(8 \mathrm{H}$, $\mathrm{m}, \mathrm{Ar}) ; \mathrm{MS}(\mathrm{m} / z, 371(17.6 \%))\left(\mathrm{M}^{+}\right)$.

2.7b 3-benzyl-5-[2-(4-methoxy-3-methylphenyl)-2oxoethyl]-2-thioxo-1,3-thiazolidin-4-one $(8 \mathrm{~b})$ : From ethanol, M.p. $142^{\circ} \mathrm{C}$. Anal. Calcd. for $\mathrm{C}_{20} \mathrm{H}_{19} \mathrm{NO}_{3} \mathrm{~S}_{2}$ (385.49): C, 62.31; H, 4.97; N, 3.63. Found: C, 62.55; H, 5.00; N, 3.58; IR (KBr, v, cm $\left.{ }^{-1}\right): 1716(\mathrm{C}=\mathrm{O}), 1664$ $(\mathrm{C}=\mathrm{O}), 1240(\mathrm{C}=\mathrm{S}) ;{ }^{1} \mathrm{H}-\mathrm{NMR}$ (DMSO- $\left.d_{6}, \delta \mathrm{ppm}\right)$ : $2.16\left(3 \mathrm{H}, \mathrm{s}, \mathrm{CH}_{3} \mathrm{Ar}\right), 3.29\left(2 \mathrm{H}, \mathrm{s}, \mathrm{CH}_{2} \mathrm{~N}\right), 3.88(3 \mathrm{H}$, $\left.\mathrm{s}, \mathrm{OCH}_{3}\right), 4.06-4.13\left(2 \mathrm{H}, \mathrm{m}, \underline{\mathrm{CH}}_{2}-\mathrm{CH}\right), 4.94-4.97(\mathrm{H}$, $\left.\mathrm{m}, \underline{\mathrm{CH}}-\mathrm{CH}_{2}\right), 7.05-7.88(8 \mathrm{H}, \mathrm{m}, \mathrm{Ar}) ; \mathrm{MS}(\mathrm{m} / \mathrm{z}, 385$ $(17.0 \%))\left(\mathrm{M}^{+}\right)$.

2.7c 5-[2-(4-methoxy-3-methylphenyl)-2-oxoethyl]-3-(4tolyl)-2-thioxo-1,3-thiazolidin-4-one $(8 \mathrm{c})$ : From ethanol, M.p. $163-165^{\circ} \mathrm{C}$. Anal. Calcd. for $\mathrm{C}_{20} \mathrm{H}_{19} \mathrm{NO}_{3} \mathrm{~S}_{2}$ (385.49): C, 62.31; H, 4.97; N, 3.63. Found: C, 62.19; $\mathrm{H}, 4.88 ; \mathrm{N}, 3.55$. IR $\left(\mathrm{KBr}, v, \mathrm{~cm}^{-1}\right): 1733(\mathrm{C}=\mathrm{O})$, $1662(\mathrm{C}=\mathrm{O}), 1251(\mathrm{C}=\mathrm{S}) ;{ }^{1} \mathrm{H}-\mathrm{NMR}\left(\mathrm{DMSO}-d_{6}, \delta\right.$ ppm): 2.20-2.37 (6H, 2s, $\left.\mathrm{CH}_{3} \mathrm{Ar}\right), 3.89\left(3 \mathrm{H}, \mathrm{s}, \mathrm{OCH}_{3}\right)$, 3.98-4.14 (2H, m, $\left.\underline{\mathrm{CH}}_{2}-\mathrm{CH}\right), 4.94-4.97$ (H, m, $\underline{\mathrm{CH}}-$ $\left.\mathrm{CH}_{2}\right), 7.07-7.90$ (7H, m, Ar); MS ( $m / z, 385$ (32.2\%)) $\left(\mathrm{M}^{+}\right)$.

\subsection{General procedure for the synthesis of 2-} substitutedamino-4-hydroxy-5-[2-(4-methoxy-3methylphenyl)-2-oxoethyl]-1,3-thiazoles (9a-d)

A mixture of the acid $(0.01 \mathrm{~mol})$; appropriate thioureas (0.01 mol); namely thiourea; phenyl thiourea; p-tolyl thiourea and/or 4-chlorophenyl thiourea and ethanol $(20 \mathrm{~mL})$ in the presence of few drops of glacial acetic acid, was refluxed for $4 \mathrm{hrs}$., the solid product that obtained after cooling was filtered, washed by ethanol and crystallized from the suitable solvent to give (9a-d).

2.8a 2-amino-4-hydroxy-5-[2-(4-methoxy-3-methylphenyl)2-oxoethyl]-1,3-thiazole (9a): From butanol, M.p. $240^{\circ} \mathrm{C}$. Anal. Calcd. for $\mathrm{C}_{13} \mathrm{H}_{14} \mathrm{~N}_{2} \mathrm{O}_{3} \mathrm{~S}$ (278.32): $\mathrm{C}$, 56.10; H, 5.07; N, 10.06. Found: C, 56.00; H, 5.00; $\mathrm{N}, 10.12$; IR $\left(\mathrm{KBr}, v, \mathrm{~cm}^{-1}\right): 3219(\mathrm{NH}), 2964(\mathrm{CH}$ aliph.), $1665(\mathrm{C}=\mathrm{O}) ;{ }^{1} \mathrm{H}-\mathrm{NMR}$ (DMSO- $\left.d_{6}, \delta \mathrm{ppm}\right)$ : 
$2.14\left(3 \mathrm{H}, \mathrm{s}, \mathrm{CH}_{3}\right), 3.29-3.45\left(2 \mathrm{H}, \mathrm{m}, \underline{\mathrm{CH}}_{2}-\mathrm{CH}\right), 3.80$ $\left(3 \mathrm{H}, \mathrm{s}, \mathrm{OCH}_{3}\right), 4.34-4.39\left(1 \mathrm{H}, \mathrm{m}, \mathrm{CH}-\mathrm{CH}_{2}\right), 7.03-7.80$ (3H, m, Ar-H), 8.76, 9.00 (2H, 2s, NH); MS ( $m / z, 278$ $(20 \%))\left(\mathrm{M}^{+}\right)$.

2.8b 2-anilino-4-hydroxy-5-[2-(4-methoxy-3-methylphenyl)-2-oxoethyl]-1,3-thiazole (9b): From butanol, M.p. $210^{\circ}$ C. Anal. Calcd. for $\mathrm{C}_{19} \mathrm{H}_{18} \mathrm{~N}_{2} \mathrm{O}_{3} \mathrm{~S}$ (354.42): C, 64.39; H, 5.12; N, 7.90. Found: C, 64.40; H, 5.00; $\mathrm{N}, 8.03$; IR $\left(\mathrm{KBr}, v, \mathrm{~cm}^{-1}\right)$ : $3260(\mathrm{NH}), 1664(\mathrm{C}=\mathrm{O})$; ${ }^{1} \mathrm{H}-\mathrm{NMR}$ (DMSO- $\left.d_{6}, \delta \mathrm{ppm}\right): 2.11\left(3 \mathrm{H}, \mathrm{s}, \mathrm{CH}_{3}\right), 3.29-$ $3.64\left(2 \mathrm{H}, \mathrm{m}, \underline{\mathrm{CH}}_{2}-\mathrm{CH}\right), 3.86\left(3 \mathrm{H}, \mathrm{s}, \mathrm{OCH}_{3}\right), 4.40-4.50$ $\left(\mathrm{H}, \mathrm{m}, \mathrm{CH}-\mathrm{CH}_{2}\right), 6.96-7.89(8 \mathrm{H}, \mathrm{m}, \mathrm{Ar}-\mathrm{H}), 11.15(\mathrm{H}$, $\mathrm{s}, \mathrm{NH}) ; \mathrm{MS}(m / z, 354(42 \%))\left(\mathrm{M}^{+}\right)$.

2.8c 4-hydroxy-5-[2-(4-methoxy-3-methylphenyl)-2oxoethyl]-2-(4-toludino)-1,3-thiazole (9c): From butanol, M.p. $225^{\circ}$ C. Anal. Calcd. for $\mathrm{C}_{20} \mathrm{H}_{20} \mathrm{~N}_{2} \mathrm{O}_{3} \mathrm{~S}$ (368.45): C, 65.20; H, 5.47; N, 7.60. Found: C, 65.51; H, 5.63; N, 7.91; IR $\left(\mathrm{KBr}, v, \mathrm{~cm}^{-1}\right): 3181(\mathrm{NH}), 1684(\mathrm{C}=\mathrm{O})$; ${ }^{1} \mathrm{H}-\mathrm{NMR}$ (DMSO- $\left.d_{6}, \delta \mathrm{ppm}\right): 2.11,2.26(6 \mathrm{H}, 2 \mathrm{~s}$, $2 \mathrm{CH}_{3}$-Ar.), 3.28-3.55 (2H, m, $\left.\mathrm{CH}_{2}-\mathrm{CH}\right), 3.86(3 \mathrm{H}, \mathrm{s}$, $\left.\mathrm{OCH}_{3}\right), 4.44-4.48\left(\mathrm{H}, \mathrm{m}, \mathrm{CH}-\mathrm{CH}_{2}\right), 6.88-7.89(7 \mathrm{H}, \mathrm{m}$, Ar-H), $11.08(1 \mathrm{H}, \mathrm{s}, \mathrm{NH})$; MS $(m / z, 368(98 \%))\left(\mathrm{M}^{+}\right)$.

2.8d 2-(4-chloroanilino)-4-hydroxy-5-[2-(4-methoxy3-methylphenyl)-2-oxoethyl]-1,3-thiazole (9d): From acetic acid, M.p. $228^{\circ} \mathrm{C}$. Anal. Calcd. for $\mathrm{C}_{19} \mathrm{H}_{17} \mathrm{ClN}_{2}$ $\mathrm{O}_{3} \mathrm{~S}$ (388.45): C, 58.69; H, 4.41; N, 7.20. Found: C, 59.06; H, 4.92; N, 6.97; IR (KBr, $\left.v, \mathrm{~cm}^{-1}\right): 3173(\mathrm{NH})$, $1667(\mathrm{C}=\mathrm{O}) ;{ }^{1} \mathrm{H}-\mathrm{NMR}\left(\mathrm{DMSO}-d_{6}, \delta \mathrm{ppm}\right): 2.17(3 \mathrm{H}$, $\left.\mathrm{s}, \mathrm{CH}_{3}\right), 3.28-3.67\left(2 \mathrm{H}, \mathrm{m}, \mathrm{CH}_{2}-\mathrm{CH}\right), 3.86(3 \mathrm{H}, \mathrm{s}$, $\left.\mathrm{OCH}_{3}\right), 4.40-4.60\left(\mathrm{H}, \mathrm{m}, \mathrm{CH}-\mathrm{CH}_{2}\right), 6.91-7.98(7 \mathrm{H}, \mathrm{m}$, Ar-H), $11.32(\mathrm{H}, \mathrm{s}, \mathrm{NH})$; MS $(m / z, 388(62 \%))\left(\mathrm{M}^{+}\right)$, $390(27 \%)\left(\mathrm{M}^{+}+2\right)$.

2.9 General procedure for the synthesis of 6-arylamino-1, 4-dihydro-3-[4-methoxy-3-methylphenyl]-1,3-thiazolo [4,5-c]pyridazines (10a-c)

To a solution of the thiazoles $(\mathbf{9 b - d})(0.01 \mathrm{~mol})$ in ethanol $(20 \mathrm{~mL})$; hydrazine hydrate $80 \%(0.015 \mathrm{~mol})$ was added and the reaction mixture was refluxed for $6 \mathrm{~h}$. The solid that separated after cooling was crystallized from suitable solvent to give the corresponding thiazolopyridazine (10a-c).

2.9a 6-anilino-1,4-dihydro-3-[4-methoxy-3-methylphenyl] -1,3-thiazolo[4,5-c]pyridazine (10a): From ethanol, M.p. $290^{\circ}$ C. Anal. Calcd. for $\mathrm{C}_{19} \mathrm{H}_{18} \mathrm{~N}_{4} \mathrm{OS}$ (350.43):
C, 65.12; H, 5.18; N, 15.99. Found: C, 65.33; H, 5.16; N, 16.11; IR (KBr, v, cm $\left.{ }^{-1}\right): 3349(\mathrm{NH}), 1601$ $(\mathrm{C}=\mathrm{N})$ or $(\mathrm{C}=\mathrm{C}) ;{ }^{1} \mathrm{H}-\mathrm{NMR}$ (DMSO- $\left.d_{6}, \delta \mathrm{ppm}\right): 2.19$ $\left(3 \mathrm{H}, \mathrm{s}, \mathrm{CH}_{3}\right), 3.24\left(2 \mathrm{H}, \mathrm{s}, \mathrm{CH}_{2}\right), 3.89\left(3 \mathrm{H}, \mathrm{s}, \mathrm{OCH}_{3}\right)$, 6.75-7.85 (8H, m, Ar-H); MS $(m / z, 350(1.3 \%))\left(\mathrm{M}^{+}\right)$.

$2.9 \mathrm{~b}$ 1,4-dihydro-3-[4-methoxy-3-methylphenyl]-6-(4toludino)-1,3-thiazolo[4,5-c]pyridazine (10b): From ethanol, M.p. $240-242^{\circ} \mathrm{C}$. Anal. Calcd. for $\mathrm{C}_{20} \mathrm{H}_{20} \mathrm{~N}_{4} \mathrm{OS}$ (364.46): C, 65.91; H, 5.53; N, 15.37. Found: C, 66.05; $\mathrm{H}, 5.76 ; \mathrm{N}, 15.29$; IR (KBr, v, $\left.\mathrm{cm}^{-1}\right): 3342(\mathrm{NH}), 1603$ $(\mathrm{C}=\mathrm{N})$ or $(\mathrm{C}=\mathrm{C})$. $)$; ${ }^{1} \mathrm{H}-\mathrm{NMR}$ (DMSO- $\left.d_{6}, \delta \mathrm{ppm}\right)$ : 2.16, $2.19\left(6 \mathrm{H}, 2 \mathrm{~s}, 2 \mathrm{CH}_{3}-\mathrm{Ar}\right), 3.33\left(2 \mathrm{H}, \mathrm{s}, \mathrm{CH}_{2}\right), 3.80$ $\left(3 \mathrm{H}, \mathrm{s}, \mathrm{OCH}_{3}\right), 6.93-7.89(7 \mathrm{H}, \mathrm{m}, \mathrm{Ar}-\mathrm{H}), 8.96(\mathrm{H}$, $\mathrm{s}, \mathrm{NH}), 13.1(\mathrm{H}, \mathrm{s}, \mathrm{NH})$; $\mathrm{MS}(\mathrm{m} / z, 363(14.7 \%))$ $\left(\mathrm{M}^{+}-\mathrm{H}\right)$.

2.9c 6-(4-chloroanilino)-1,4-dihydro-3-[4-methoxy-3methylphenyl]-1,3-thiazolo[4,5-c]pyridazine (10c): From butanol, M.p. 256-258 ${ }^{\circ}$ C. Anal. Calcd. for $\mathrm{C}_{19} \mathrm{H}_{17}$ $\mathrm{ClN}_{4} \mathrm{OS}$ (384.88): C, 59.29; H, 4.45; N, 14.56. Found: C, 59.00; H, 4.56; N, 14.48; IR (KBr, v, cm $\left.{ }^{-1}\right): 3331$ $(\mathrm{NH}), 1601(\mathrm{C}=\mathrm{N})$ or $(\mathrm{C}=\mathrm{C})$; ${ }^{1} \mathrm{H}-\mathrm{NMR}$ (DMSO$\left.d_{6}, \delta \mathrm{ppm}\right): 2.16\left(3 \mathrm{H}, \mathrm{s}, \mathrm{CH}_{3}\right), 3.30\left(2 \mathrm{H}, \mathrm{s}, \mathrm{CH}_{2}\right)$, $3.80\left(3 \mathrm{H}, \mathrm{s}, \mathrm{OCH}_{3}\right), 6.93-7.55(7 \mathrm{H}, \mathrm{m}, \mathrm{Ar}-\mathrm{H}), 9.2$ $(\mathrm{H}, \mathrm{s}, \mathrm{NH}), 13.2(\mathrm{H}, \mathrm{s}, \mathrm{NH}) ; \mathrm{MS}(m / z, 384(4.5 \%))$ $\left(\mathrm{M}^{+}\right)$.

2.10 Synthesis of 1,4-dihydro-3-[4-methoxy-3-methylphenyl]-1-phenyl-6-(4-toludino)-1,3-thiazolo[4,5-c] pyridazine (11)

A mixture of 4-hydroxy-5-[2-(4-methoxy-3-methylphenyl)-2-oxoethyl]-2-(4-toludino)-1,3-thiazole (9b) $(0.005 \mathrm{~mol})$ and phenylhydrazine hydrochloride $(0.006$ mol) in pyridine $(5 \mathrm{~mL})$ was heated under reflux for 6 hrs. the solid separated after evaporation of the most of the solvent and cooling, was washed by benzene and crystallized from the proper solvent to give (11) with M.p. $316^{\circ}$ C. Anal. Calcd. for $\mathrm{C}_{26} \mathrm{H}_{24} \mathrm{~N}_{4} \mathrm{OS}$ (440.56); C, 70.88; H, 5.49; N, 12.72; Found: C, 71.32; H, 5.42; N, 12.66; IR (KBr, v, $\left.\mathrm{cm}^{-1}\right): 3018$ (CH-Ar), 283902918 (CH aliph.), $1620(\mathrm{C}=\mathrm{C}) ;{ }^{1} \mathrm{H}-\mathrm{NMR}$ (DMSO- $d_{6}$, $\delta \mathrm{ppm}): 2.23$ and $2.25\left(6 \mathrm{H}, 2 \mathrm{~s}, 2 \mathrm{CH}_{3}-\mathrm{Ar}\right), 2.36(2 \mathrm{H}, \mathrm{s}$, $\left.\mathrm{CH}_{2}\right), 3.89$ (3H, s, $\left.\mathrm{OCH}_{3}\right), 7.20-8.03(12 \mathrm{H}, \mathrm{m}, \mathrm{Ar}-\mathrm{H})$ $9.23(\mathrm{H}, \mathrm{s}, \mathrm{NH})$; $\mathrm{MS}(\mathrm{m} / z, 440(10 \%))\left(\mathrm{M}^{+}\right)$.

\subsection{Synthesis of acetyl derivatives of thiazoles (12a-d)}

A solution of thiazoles (9a-d) (1 gm) in acetic anhydride $(5 \mathrm{~mL})$ was refluxed for $4 \mathrm{~h}$. The solid 
product obtained, after evaporation of most of the solvent and cooling, was filtered off, washed with ethanol and crystallized from the proper solvent to give (12a-d).

2.11a 4-acetoxy-2-amino-5-[2-(4-methoxy-3-methylphenyl)-2-oxoethyl]-1,3-thiazole (12a): from butanol, M.p. $205^{\circ} \mathrm{C}$. Anal. Calcd. for $\mathrm{C}_{15} \mathrm{H}_{16} \mathrm{~N}_{2} \mathrm{O}_{4} \mathrm{~S}$ (320.36): C, 56.24; H, 5.03; N, 8.74. Found: C, 56.47; H, 4.88; N, 8.91; IR (KBr, v, $\left.\mathrm{cm}^{-1}\right)$ : $3439(\mathrm{OH}), 3237(\mathrm{NH})$, $1779\left(\mathrm{CO}-\mathrm{CH}_{3}\right), 1672$ (CO-Ar); ${ }^{1} \mathrm{H}-\mathrm{NMR}$ (DMSO-d $d_{6}$, $\delta \mathrm{ppm}): 2.17\left(3 \mathrm{H}, \mathrm{s}, \mathrm{CH}_{3} \mathrm{Ar}\right), 2.38\left(2 \mathrm{H}, \mathrm{s}, \mathrm{CH}_{2}\right), 2.43$ $\left(3 \mathrm{H}, \mathrm{s}, \mathrm{CH}_{3} \mathrm{CO}\right), 3.80\left(3 \mathrm{H}, \mathrm{s}, \mathrm{OCH}_{3}\right), 6.81-7.42(3 \mathrm{H}$, $\mathrm{m}, \operatorname{Ar}-\mathrm{H}), 12.34(\mathrm{H}, \mathrm{s}, \mathrm{NH}) ; \mathrm{MS}(m / z, 320(100 \%))$ $\left(\mathrm{M}^{+}\right)$.

2.11b 4-acetoxy-2-anilino-5-[2-(4-methoxy-3-methylphenyl)-2-oxoethyl]-1,3-thiazole (12b): From chloroform, M.p. $275^{\circ} \mathrm{C}$. Anal. Calcd. for $\mathrm{C}_{21} \mathrm{H}_{20} \mathrm{~N}_{2} \mathrm{O}_{4} \mathrm{~S}$ (396.46): C, 63.62; H, 5.08; N, 7.07. Found: C, 63.90; $\mathrm{H}, 5.21 ; \mathrm{N}, 6.66 ; \mathrm{IR}\left(\mathrm{KBr}, v, \mathrm{~cm}^{-1}\right): 1781\left(\mathrm{CO}-\mathrm{CH}_{3}\right)$, 1679 (CO-Ar); ${ }^{1} \mathrm{H}-\mathrm{NMR}$ (DMSO- $d_{6}, \delta$ ppm): $2.17(2 \mathrm{H}$, s, $\left.\mathrm{CH}_{2}\right), 2.27\left(3 \mathrm{H}, \mathrm{s}, \mathrm{CH}_{3} \mathrm{Ar}\right), 2.51\left(3 \mathrm{H}, \mathrm{s}, \mathrm{CH}_{3}-\mathrm{CO}\right)$, $3.87\left(3 \mathrm{H}, \mathrm{s}, \mathrm{OCH}_{3}\right), 7.05-7.88(8 \mathrm{H}, \mathrm{m}, \mathrm{Ar}-\mathrm{H}) ; \mathrm{MS}$ $(\mathrm{m} / \mathrm{z}, 396(4.4 \%))\left(\mathbf{M}^{+}\right)$.

2.11c 4-acetoxy-2-(4-toludino)-5-[2-(4-methoxy-3-methylphenyl)-2-oxoethyl]-1,3-thiazole (12c): from ethanol, M.p. $215^{\circ} \mathrm{C}$. Anal. Calcd. for $\mathrm{C}_{22} \mathrm{H}_{22} \mathrm{~N}_{2} \mathrm{O}_{4} \mathrm{~S}$ (410.48): C, 64.37; H, 5.40; N, 6.82. Found: C, 64.20; H, 5.02; $\mathrm{N}, 6.77$; IR $\left(\mathrm{KBr}, v, \mathrm{~cm}^{-1}\right)$ : $1768\left(\mathrm{CO}-\mathrm{CH}_{3}\right), 1672$ (CO-Ar); ${ }^{1} \mathrm{H}-\mathrm{NMR}$ (DMSO- $\left.d_{6}, \delta \mathrm{ppm}\right): 2.18,2.28(6 \mathrm{H}$, $\left.2 \mathrm{~s}, 2 \mathrm{CH}_{3}-\mathrm{Ar}\right), 2.38\left(3 \mathrm{H}, \mathrm{s}, \mathrm{CH}_{3}-\mathrm{C}=\mathrm{O}\right), 2.48(2 \mathrm{H}, \mathrm{s}$, $\left.\mathrm{CH}_{2}\right), 3.81\left(3 \mathrm{H}, \mathrm{s}, \mathrm{OCH}_{3}\right), 6.72-7.41(7 \mathrm{H}, \mathrm{m}, \mathrm{Ar}-\mathrm{H})$; MS $(m / z: 410(16.7 \%))\left(\mathbf{M}^{+}\right)$.

2.11d 4-acetoxy-2-(4-chloroanilino)-5-[2-(4-methoxy3-methylphenyl)-2-oxoethyl]-1,3-thiazole (12d): From butanol, M.p. $208^{\circ} \mathrm{C}$. Anal. Calcd. for $\mathrm{C}_{21} \mathrm{H}_{19} \mathrm{ClN}_{2} \mathrm{O}_{4} \mathrm{~S}$ (430.90): C, 58.54; H, 4.44; N, 6.50. Found: C, 58.55; $\mathrm{H}, 4.44 ; \mathrm{N}, 6.38$; IR (KBr, $\left.v, \mathrm{~cm}^{-1}\right): 1768\left(\mathrm{CO}-\mathrm{CH}_{3}\right)$, 1672 (CO-Ar); ${ }^{1} \mathrm{H}-\mathrm{NMR}$ (DMSO- $d_{6}, \delta$ ppm): $1.98(3 \mathrm{H}$, s, $\left.\mathrm{CH}_{3}-\mathrm{Ar}\right), 2.20\left(2 \mathrm{H}, \mathrm{s}, \mathrm{CH}_{2}\right), 2.28\left(3 \mathrm{H}, \mathrm{s}, \mathrm{CH}_{3}-\mathrm{CO}\right)$, $3.81\left(3 \mathrm{H}, \mathrm{s}, \mathrm{OCH}_{3}\right), 6.73-7.64(7 \mathrm{H}, \mathrm{m}, \mathrm{Ar}-\mathrm{H})$; MS $(\mathrm{m} / \mathrm{z}, 430(60.6 \%))\left(\mathbf{M}^{+}\right)$.

2.12 Synthesis of 2-substitutedamino-4-hydroxy-5-[2(4-methoxy-3-methylphenyl)-1-bromo-2-oxoethyl]-1,3thiazoles (13a-b)

To a solution of thiazoles $(\mathbf{9 b})$ or $(\mathbf{9 d})(0.005 \mathrm{~mol})$ in acetic acid $(40 \mathrm{~mL})$, a bromine solution $(0.005 \mathrm{~mol})$ in acetic acid $(10 \mathrm{~mL})$ was added slowly with stirring.
After addition was complete, the mixture was gently warmed until $\mathrm{HBr}$ gas evolution ceased. The precipitated solid was filtered off, washed with ethanol, air dried and crystallized from the proper solvent to give (13a-b).

2.12a 2-anilino-4-hydroxy-5-[2-(4-methoxy-3-methylphenyl)-1-bromo-2-oxoethyl]-1,3-thiazole (13a): from ethanol, M.p. 250-252 ${ }^{\circ}$ C. Anal. Calcd. for $\mathrm{C}_{19} \mathrm{H}_{17}$ $\mathrm{BrN}_{2} \mathrm{O}_{3} \mathrm{~S}$ (433.31): C, 52.67; H, 3.95; N, 6.46. Found: C, 52.49; H, 3.88; N, 6.52; IR (KBr, v, $\left.\mathrm{cm}^{-1}\right): 3254$ $(\mathrm{NH}), 1696$ (CO-Ar); ${ }^{1} \mathrm{H}-\mathrm{NMR}$ (DMSO- $d_{6}, \delta \mathrm{ppm}$ ): 2.19 (3H, s, $\left.\mathrm{CH}_{3}-\mathrm{Ar}\right), 3.32-3.45$ (2H, m, CH-CH), 3.89 $\left(3 \mathrm{H}, \mathrm{s}, \mathrm{OCH}_{3}\right), 7.04-8.10(8 \mathrm{H}, \mathrm{m}, \mathrm{Ar}-\mathrm{H}), 11.76(\mathrm{H}, \mathrm{s}$, $\mathrm{NH})$; $\mathrm{MS}(\mathrm{m} / z, 433(1.0 \%))\left(\mathbf{M}^{+}\right)$.

2.12b 2-(4-chloroanilino)-4-hydroxy-5-[2-(4-methoxy3-methylphenyl)-1-bromo-2-oxoethyl]-1,3-thiazole (13b): From ethanol, M.p. 278-280 ${ }^{\circ}$ C. Anal. Calcd. for $\mathrm{C}_{19} \mathrm{H}_{16} \mathrm{BrClN}_{2} \mathrm{O}_{3} \mathrm{~S}$ (467.76): C, 48.79; H, 3.45; N, 5.99. Found: C, 49.20; H, 3.55; N, 6.10; IR (KBr, v, $\left.\mathrm{cm}^{-1}\right)$ : $3253(\mathrm{NH}), 1682$ (CO-Ar); ${ }^{1} \mathrm{H}-\mathrm{NMR}$ (DMSO- $d_{6}, \delta$ ppm): $2.18\left(3 \mathrm{H}, \mathrm{s}, \mathrm{CH}_{3}-\mathrm{Ar}\right), 3.53-3.56(2 \mathrm{H}, \mathrm{m}, \mathrm{CH}-$ $\mathrm{CH}), 3.89\left(3 \mathrm{H}, \mathrm{s}, \mathrm{OCH}_{3}\right), 7.39-8.10(7 \mathrm{H}, \mathrm{m}, \mathrm{Ar}-\mathrm{H})$, $11.87(\mathrm{H}, \mathrm{s}, \mathrm{NH})$; $\mathrm{MS}(m / z, 386(18.3 \%))\left(\mathrm{M}^{+}-\mathrm{HBr}\right)$.

\section{Results and Discussion}

The synthetic routes of the prepared derivatives are shown in schemes 1,2 and 3. The key compound, 4-[4methoxy-3-methylphenyl]-4-oxobutenoic acid (1), was synthesized in good yield by Friedel-Crafts acylation of $o$-cresyl methyl ether with maleic anhydride according to reported method. ${ }^{13}$

The aza-Michael addition with primary and secondary amines was carried out by the treatment of the acid (1) with amines in dry benzene in good yields with the formation of the corresponding $\alpha$-substituted amino acids (2a-e). These compounds were formed through the attack of the amino group on the electron-deficient olefinic carbon atom, $\alpha$ - to the carboxyl group. IR spectra showed prominent bands for $\mathrm{OH}$ groups at 3436$3438 \mathrm{~cm}^{-1}$ and for $\mathrm{C}=\mathrm{O}$ groups at $1668-1675 \mathrm{~cm}^{-1}$. ${ }^{1} \mathrm{H}-\mathrm{NMR}$ spectra the aromatic protons were observed at expected ppm's. The signals for $\mathrm{OH}$ groups were observed as merged with the signals of aromatic protons in aromatic region.

Surprisingly, when the reaction of the $\alpha$-substituted amino acids (2) with hydrazine was carried out in refluxing ethanol, we unexpectedly obtained 6(4-methoxy-3-methylphenyl)-3(2H)-pyridazinone (3) 


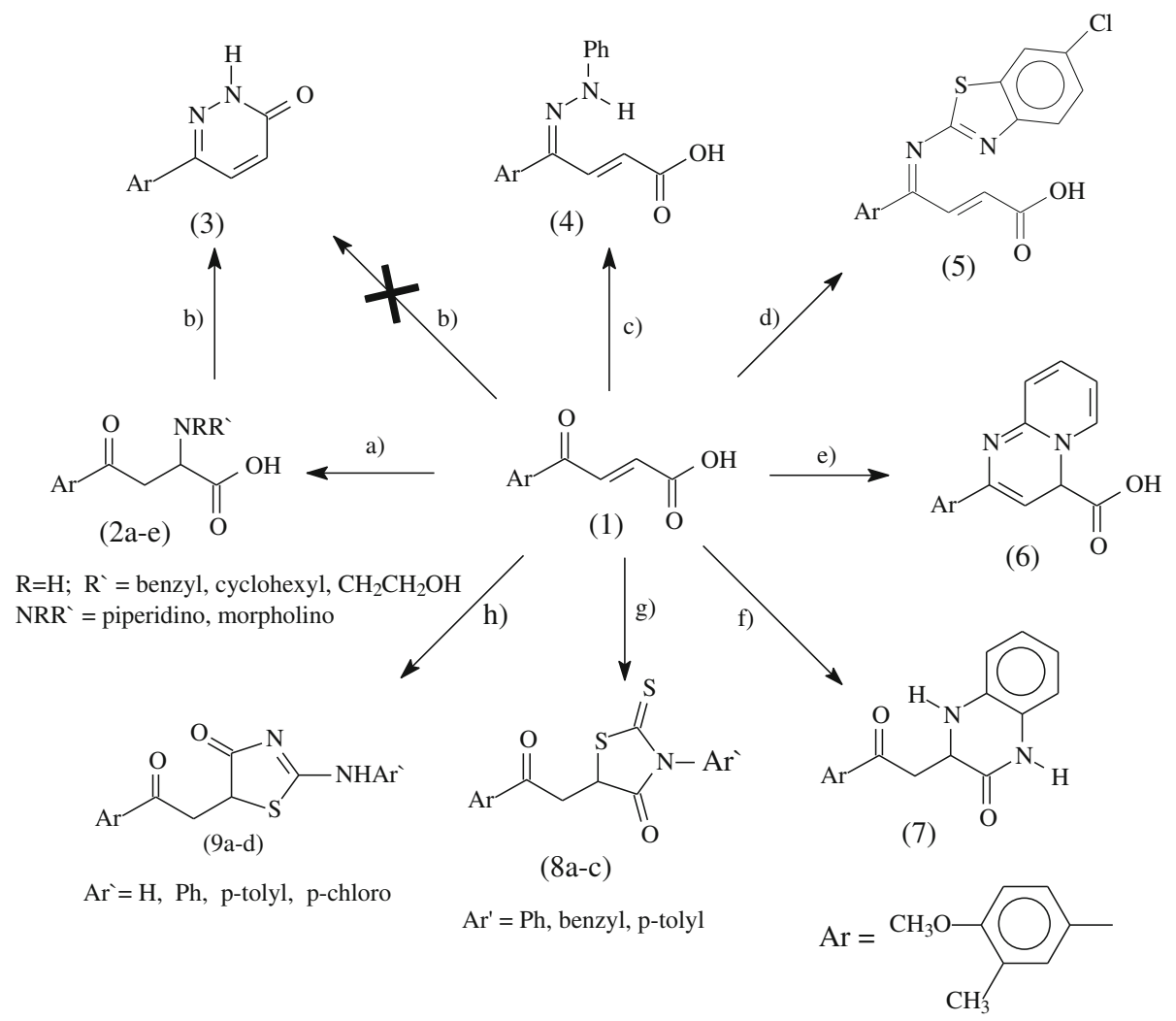

\section{Reaction conditions}

a) amines, dry benzen; b) hydrazine, ethanol, reflux; c) phenylhydrazine, ethanol, reflux;

d) 2-amino-6-chlorobenzothiazole, ethanol, reflux; e) 2-aminopyridine, ethanol, reflux;

f) o-phenylene diamine, ethanol, reflux; g) ammonium aryl dithiocarbamate, $\mathrm{HCl}$

h) thioureas, ethanol and $\mathrm{AcOH}$, reflux;

Scheme 1. Reactions of Oxobutenoic acid derivative with different nucleophiles.

(Scheme 2) instead of 4-alkylamino dihydropyridazinone. In the presence of hydrazine hydrate the acid (2) underwent cyclization with the formation of 4alkylamino dihydropyridazinone followed by the fission of the benzylamino group in position 4 and aromatization of the pyridazine ring. The proposed structure of this compound was supported spectral data and by the identity of its m.p. with that of an authentic sample prepared by us ${ }^{14}$ from the reaction of the corresponding

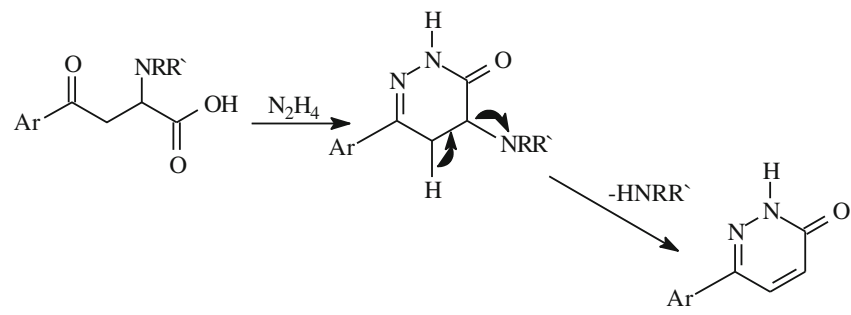<smiles>N#CC1CCC(=O)NN1</smiles>

(3)

Scheme 2. Formation of 6-(4-methoxy-3-methylphenyl)$3(2 \mathrm{H})$-pyridazinone.

4-(4-methoxy-3-methylphenyl)-4-oxobutanoic acid with hydrazine hydrate and treating the resulting dihydropyridazinone with bromine.

Attempts to obtain a solid product through the reaction of the acid (1) with hydrazine hydrate under different conditions were failed. However, when the acid was treated with phenylhydrazine in ethanol afforded the corresponding 4-[4-methoxy-3-methylphenyl]-4oxobutenoic acid phenyl hydrazone (4). The IR showed bands in the region of $3500 \mathrm{~cm}^{-1}, 3294 \mathrm{~cm}^{-1}$ and 1679 $\mathrm{cm}^{-1}$ which are assigned to the vibrations of the $\mathrm{OH}$, $\mathrm{NH}$ and $\mathrm{C}=\mathrm{O}$ groups, respectively.

In similar way, when the acid (1) was submitted to react with 2-amino-6-chlorobenzothiazole in boiling ethanol it afforded 4-[6-chlorobenzothiazol-2-ylimino]4-[4-methoxy-3-methylphenyl]-4-oxobutenoic (5). The reaction takes place via the condensation reaction between the amino and keto groups with the formation of the corresponding Schiff base.

The treatment of the acid (1) with 2-aminopyridine in boiling ethanol resulted in the formation of pyridopyrimidine derivative (6). The formation of compound (6) was assumed to proceed via the addition of the amine 


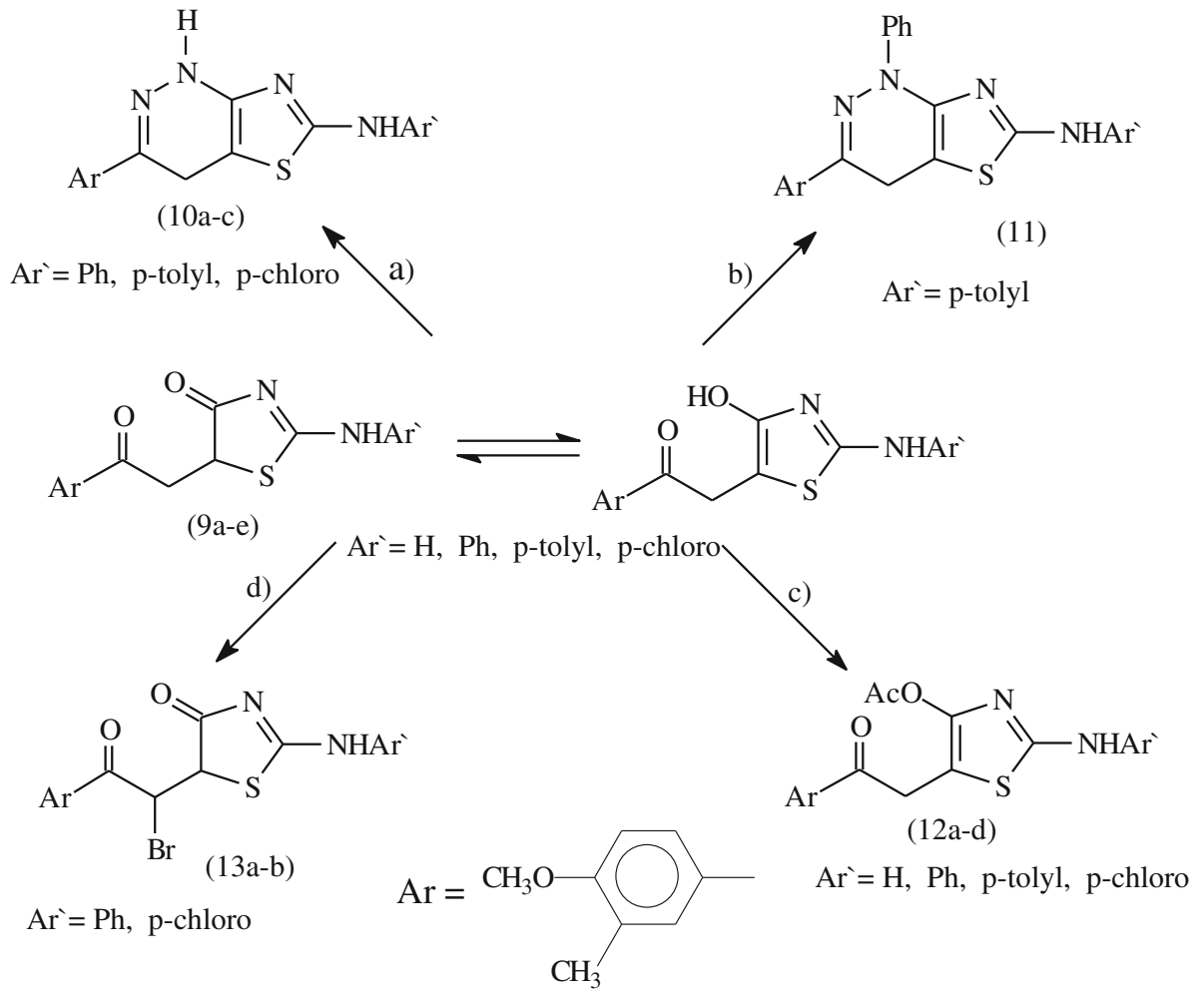

\section{Reaction conditions}

a) hydrazine, ethanol, reflux; b) phenylhydrazine hydrochloride, pyridine, reflux;

c) acetic anhydrid, reflux; d) bromine, acetic acid, stirring

Scheme 3. Reactions of 2-aminothiazole derivatives.

to the double bond followed by cyclocondensation with the keto group.

The interaction of the acid (1) with o-phenylene diamine in refluxing ethanol afforded the corresponding quinoxaline (7). The ${ }^{1} \mathrm{H}-\mathrm{NMR}$ showed multiplets for the methylene and methine groups protons $(\delta=3.20$ $3.88)$, a signal for the amino group $(\delta=5.88 \mathrm{ppm})$, a multiplet for the aromatic protons $(\delta=6.06-7.89)$ and also a downfield singlet ( $\delta=10.2 \mathrm{ppm}$ ) which is assigned to the amido $\mathrm{NH}$ proton.

When the acid (1) was allowed to react with ammonium aryldithiocarbamates in ethanol in the presence of hydrochloric acid gave the corresponding 2-thioxo-1,3thiazolidin-4-one derivatives ( $\mathbf{8 a - c}$ ) in good yield. The structures of these compounds were confirmed by the absence of the absorption bands corresponding to the hydroxyl group in their IR spectra and the appearance of tow multiplets in the aliphatic regions corresponding to the methylene $(\delta=3.77-4.14)$ and methine $(\delta=4.93-4.97)$ groups in their ${ }^{1} \mathrm{H}-\mathrm{NMR}$ spectra.

Similarly, the 2-substitutedamino-4-hydroxy-5-[2(4-methoxy-3-methylphenyl)-2-oxoethyl]-1,3-thiazoles $(9 \mathbf{a}-\mathbf{c})$ were obtained from the reaction of the acid (1) with the appropriate thiourea derivatives in boiling ethanol containing catalytic amount of glacial acetic acid.

The 2-aminothiazole derivatives (9a-c) were considered as key starting material in which they reacted with various reagents (Scheme 2). Thus, the treatment of $(\mathbf{9 a - c )}$ with hydrazine hydrate in refluxing ethanol or phenylhydrazine hydrochloride in pyridine afforded the corresponding thiazolopyridazine derivatives (10a-c) and (11), respectively. The structures of these compounds were confirmed by the absence of the characteristic absorption bands corresponding to the carbonyl group in their IR spectra.

The acetylation of the aminothiazolons $(\mathbf{9 a}-\mathbf{c})$ was carried out by boiling them with acetic anhydride giving the 5-acetoxy-2-substitutedamino-thiazoles (12a-c). The IR spectra of these compounds showed the appearance of new absorption bands in the region 1768-1781 $\mathrm{cm}^{-1}$ corresponding to the acetate group which confirmed that the acetylation occurred at the hydroxyl groups and the appearance of new singlets in the aliphatic regions corresponding to the acetate groups $(\delta=2.28-2.51)$ in their ${ }^{1} \mathrm{H}-\mathrm{NMR}$ spectra.

The 2-aminothiazolone derivatives $(\mathbf{9 b}-\mathbf{c})$ underwent bromination upon treatment with bromine in acetic 
Table 1. The anti-bacterial effects of the synthesized compounds.

\begin{tabular}{|c|c|c|c|c|c|c|}
\hline \multirow[t]{2}{*}{ Compound No. } & \multicolumn{3}{|c|}{ Gram negative bacteria } & \multicolumn{3}{|c|}{ Gram postive bacteria } \\
\hline & $\begin{array}{c}\text { Pseudomonas } \\
\text { aeruginosa }\end{array}$ & $\begin{array}{c}\text { Serratia } \\
\text { sp. }\end{array}$ & $\begin{array}{l}\text { Escherichia } \\
\text { coli }\end{array}$ & $\begin{array}{l}\text { Micrococcus } \\
\quad \text { luteus }\end{array}$ & $\begin{array}{l}\text { Bacillus } \\
\text { subtilis }\end{array}$ & $\begin{array}{c}\text { Staphylococcus } \\
\text { aureus }\end{array}$ \\
\hline $2 \mathrm{a}$ & + & + & - & + & + & ++ \\
\hline $2 b$ & - & + & - & + & + & + \\
\hline $2 \mathrm{c}$ & ++ & + & + & + & + & +++ \\
\hline $2 \mathrm{~d}$ & + & + & + & - & + & +++ \\
\hline $2 \mathrm{e}$ & + & + & - & - & + & +++ \\
\hline 3 & + & + & - & + & + & ++ \\
\hline 4 & - & - & - & + & - & ++ \\
\hline 5 & + & + & - & + & + & ++ \\
\hline 6 & + & + & + & + & + & ++ \\
\hline 7 & - & - & - & - & - & - \\
\hline $8 \mathrm{a}$ & + & - & - & - & - & - \\
\hline $8 b$ & ++ & - & + & + & - & - \\
\hline $8 \mathrm{c}$ & - & - & - & - & - & - \\
\hline $9 \mathrm{a}$ & + & + & - & - & - & - \\
\hline $9 \mathrm{~b}$ & + & + & - & - & - & - \\
\hline $9 \mathrm{c}$ & + & + & - & - & - & - \\
\hline $9 \mathrm{~d}$ & + & + & - & - & - & + \\
\hline $10 \mathrm{a}$ & + & - & - & +++ & + & - \\
\hline $10 \mathrm{~b}$ & ++ & - & - & - & - & - \\
\hline $10 \mathrm{c}$ & - & - & - & + & - & - \\
\hline 11 & + & - & - & - & - & - \\
\hline $12 \mathrm{a}$ & + & - & - & - & - & + \\
\hline $12 \mathrm{~b}$ & + & + & + & - & - & + \\
\hline $12 c$ & + & - & + & - & - & + \\
\hline $12 \mathrm{~d}$ & + & - & - & + & + & - \\
\hline $13 a$ & - & - & - & - & - & - \\
\hline $13 b$ & + & - & - & - & - & - \\
\hline
\end{tabular}

Solvent: DMSO, $\mathrm{C}=1 \mathrm{mg} / \mathrm{ml} ;(-)=$ no activity; $(+)=$ mild activity $($ diameter $7-10 \mathrm{~mm})$; $(++)=$ moderate $($ diameter $10-17 \mathrm{~mm}) ;(+++)=$ high activity $($ diameter $18-22 \mathrm{~mm})$.

acid affording the 2-substituted amino-4-hydroxy-5[2-(4-methoxy-3-methylphenyl)-1-bromo-2-oxoethyl]1,3-thiazoles (13a-b).

\subsection{Biological activity}

Using paper disk agar diffusion technique ${ }^{15}$ all the newly synthesized compounds were tested in-vitro for anti-bacterial activity against strains of bacteria such as
Bacillus subtilis, Straphylocouus aureus, Micrococcus luteus, Escherichia coli, Serratia sp. and Pseudomonas aeruginosa. The compounds were tested at $1 \mathrm{mg} / \mathrm{mL}$ concentration and the activity was determined by measuring Zone of inhibition. The screening indicated that some of the synthesized compounds exhibited antibacterial activities against one or the other type of bacteria (table 1). We expect that our results may be helpful in understanding the minimum structural requirements for these compounds acting as antibacterial agents. So,

Table 2. MIC's as $(\mu \mathrm{g} / \mathrm{ml})$ of a few newly synthesized compound.

\begin{tabular}{lccccc}
\hline Compound No & \multicolumn{2}{c}{ Gram positive bacteria } & & \multicolumn{2}{c}{ Gram negative bacteria } \\
\cline { 2 - 3 } & $\begin{array}{c}\text { Pseudomonas } \\
\text { aeruginosa }\end{array}$ & $\begin{array}{c}\text { Serratia } \\
\text { sp. }\end{array}$ & & $\begin{array}{c}\text { Bacillus } \\
\text { subtilis }\end{array}$ & $\begin{array}{c}\text { Staphylococcus } \\
\text { aureus }\end{array}$ \\
\hline $2 \mathrm{a}$ & 250 & 125 & 125 & 125 \\
$2 \mathrm{~d}$ & 125 & 125 & 250 & 125 \\
$2 \mathrm{e}$ & 500 & 500 & 125 & 125 \\
5 & 500 & 125 & 125 & 125 \\
6 & 125 & $<125$ & 125 & $<125$ \\
\hline
\end{tabular}


further investigation was tested to calculate MIC's as $(\mu \mathrm{g} / \mathrm{ml})$ of those active compounds (table 2). The series of amino acid derivatives (2a-e) showed higher activity than the other compounds. The thiazole derivatives (9a-d) showed activity against Gram negative bacteria only (Pseudomonas aeruginosa and Serratia sp.). Actylation of these compound extents the activity to include also Staphylococcus aureus. While condensation with phenylhdrazine or addition to bromine lead to decrease its activity.

\section{Conclusion}

We have shown that different heterocyclic compounds have been synthesized from the reaction of 4-[4methoxy-3-methylphenyl]-4-oxobutenoic acid with different nucleophiles. Compounds which retain the carboxyl group (the series of amino acid, benzothiazole and pyridipyrimidine derivatives) showed highest and broad spectrum anti-bacterial activity compared to other compounds which lose the carboxyl group.

\section{Supplementary Information}

IR, NMR and mass spectra of compounds $2 \mathrm{a}, 2 \mathrm{~b}, 2 \mathrm{c}$, 2d, 2e, 3, 4, 5, 6, 7, 8a, 8b, 8c, 9a, 9b, 9c, 9d, 10a, 10b, $10 \mathrm{c}, 11,12 \mathrm{a}, 12 \mathrm{~b}, 12 \mathrm{c}, 12 \mathrm{~d}, 13 \mathrm{a}$ and $13 \mathrm{~b}$ are shown in Figures $\mathrm{S} 1$ to $\mathrm{S} 78$.

\section{References}

1. Kirchner F K, Bailey J H and Cavallito C J 1949 J. Amer. Chem. Soc. 711210

2. Dal Pozzo A, Acquasaliante M, Donezzeli G, De M aria $P$ and Nicoli C 1987 J. Med. Chem. 301674

3. Bowden K, Dal Pozzo A and Duah C K 1990 J. Chem. Res. Synopses 2801

4. Teichert A, Lubken T, Schmidt J, Porzel A, Arnold N and Wessjohann L 2005 Z. Naturforsch. A. 6025

5. Bianchi M, Butti A, Christidis Y, Perronnet J, Barzaghi F, Cesana R and Nencioni A 1988 Eur. J. Med. Chem. 2345

6. Giordani A, Pevarello P, Speciale C and Varasi M 2000 US Pat. 6048896

7. Juranić Z, Stevović Lj, Drakulić B, Stanojković T, Radulović S and Juranić I 1999 J. Serb. Chem. Soc. 64 505

8. Drakulić B J, Stanojković T P, Zizak Z S and Dabović M M 2011 Eur. J. Med. Chem. 463265

9. Kolos N N and Beryozkina T 2005 Chem. of Heterocyclic Comp. 41 (11) 1432

10. Jakubec P, Berkeš D, Šiška R, Gardianová $M$ and Považanec F 2006 Tetrahedron: Asymmetry 171629

11. Kolos N N, Kovalenko L U and Borovskoy V A 2011 Chem. of Heterocyclic Comp. 47 (8) 983

12. El-Hashash M A, El-Sawy A A, Eissa A M F and Sallam M S 2009 Journal of the Korean Chemical Society 53 (3) 308

13. Papa D, Schwenk E, Villani F and Klingsberg E 1948 J. Am. Chem. Soc. 703356

14. Soliman M H A and El-Sakka S S 2010 Afinidad 548 316

15. Cooper K E and Kavanagh E E 1972 In Analytical Microbiology Vol. 2 (New York: Academic Press) p 13 